\title{
Associations between adipocytokines and severity of cardiovascular autonomic neuropathy in well- controlled type 2 diabetes and prediabetes
}

\section{Yun-Ru Lai}

Chang Gung Memorial Hospital Kaohsiung Branch

\section{Wen-Chan Chiu}

Chang Gung Memorial Hospital Kaohsiung Branch

\section{Ben-Chung Cheng}

Chang Gung Memorial Hospital Kaohsiung Branch

Jung-Fu Chen

Chang Gung Memorial Hospital Kaohsiung Branch

Nai-Wen Tsai

Chang Gung Memorial Hospital Kaohsiung Branch

Chih-Cheng Huang

Chang Gung Memorial Hospital Kaohsiung Branch

Cheng-Hsien Lu ( $\nabla$ chlu99@ms44.url.com.tw)

Chang Gung Memorial Hospital Kaohsiung Branch https://orcid.org/0000-0002-5716-3930

\section{Original investigation}

Keywords: Cardiovascular autonomic neuropathy, Composite autonomic scoring scale, Cardiac autonomic reflex tests, leptin, type 2 diabetes and prediabetes

Posted Date: May 15th, 2020

DOI: https://doi.org/10.21203/rs.3.rs-27650/v1

License: (c) (i) This work is licensed under a Creative Commons Attribution 4.0 International License. Read Full License 


\section{Abstract \\ Background}

It is thought that adipocytokines contribute to autonomic dysfunction and cardiovascular risks in type 1 and type 2 diabetes mellitus (T1DM and T2DM, respectively). We aimed to identify adipocytokines that are associated with the severity of cardiovascular autonomic neuropathy (CAN) in patients with wellcontrolled T2DM and prediabetes.

\section{Methods}

The complete cardiovascular autonomic function and biomarkers were assessed for each patient. The severity of CAN was assessed using both the composite autonomic scoring scale (CASS) and the cardiac autonomic reflex tests (CARTs) score. Biomarkers included adipocytokines (leptin, chemerin, adiponectin, and vaspin), oxidative stress (thiols and thiobarbituric acid-reactive substance [TBARS]), endothelial dysfunction (sICAM-1 and SVCAM-1), and insulin resistance (triglyceride/high-density lipoprotein cholesterol [HDL-C] ratio and homeostasis model assessment of insulin resistance [HOMA-IR] index).

\section{Results}

A total of 107 patients were included in this study: 90 with diabetes and 17 with prediabetes. Stepwise logistic regression showed that diabetes duration and leptin level were independently associated with the presence of CAN. Leptin level was positively correlated with body mass index $(r=0.588, P<0.0001)$, waist circumference $(r=0.504, P<0.0001)$, urinary albumin-to-creatinine ratio $(r=0.351, P=0.001)$, hs-CRP $(r=$ $0.291, P=0.006)$, TBARS $(r=0.259, P=0.008)$, triglyceride/HDL-C ratio $(r=0.346, P<0.0001)$, and HOMAIR index $(r=0.304, P=0.008)$ but negatively correlated with thiols $(r=-0.25, P=0.011)$ level. The leptin level was strongly associated with the CASS $(r=0.36, P<0.0001)$ and CARTs $(r=0.356, P<0.0001)$ scores. Stepwise linear regression analysis also showed that the leptin level was significantly associated with the CASS and CARTs scores, respectively.

\section{Conclusions}

As the leptin level is considered a prognostic factor in CAN, a longitudinal study is needed to confirm the association between a decrease in obesity and reduced leptin levels and CAN progression.

\section{Background}

The prevalence of obesity has increased worldwide over the past several decades to become a global health problem, and it promotes an increased risk of multiple serious conditions[1, 2], including type 2 
diabetes (T2DM), cardiovascular diseases, nonalcoholic fatty liver disease, chronic kidney disease, and different types of cancer[3].

Currently, adipose tissue is recognized as a complex and dynamic endocrine organ in the body that not only stores energy, but also regulates energy homeostasis, cellular reactions, and metabolic homeostasis[4]. The adipocytes are metabolically active and potent secretory cells, capable of releasing many adipocytokines, involved in the regulation of appetite, inflammatory and immune functions, glucose and lipid metabolism, long-term energy balance, insulin sensitivity of insulin responsive tissues, cardiovascular homeostasis and reproduction, and other important biological and physiological functions[5]. Leptin is an adipocyte-secreted hormone with a key role in energy homeostasis, and it can also stimulate the secretion of several cytokines via inflammatory cells. Additionally, serum leptin levels were positively correlated with insulin resistance (IR)[6]. Adiponectin is another adipocyte-secreted hormone, which modulates several metabolic processes, including glucose regulation and fatty acid oxidation. It has a protective role against IR and anti-inflammatory activity and seems to protect against metabolic syndrome (MetS)[7]. Chemerin is an adipokine that has a crucial role in adipocyte differentiation and development, as well as in glucose and lipid metabolism[8]. Meanwhile, vaspin is an adipokine expressed in visceral white adipose tissue, and abnormalities associated with this protein are associated with the development of metabolic and glucose intolerance and atherosclerosis[9].

The pathophysiological mechanism of cardiovascular autonomic neuropathy (CAN) is multifactorial, and there is sufficient evidence that it may precede diabetes mellitus (DM)[10]. MetS is associated with multiple risk factors, including central obesity that may increase the risk of cardiovascular events in individuals with type 2 DM[11]. MetS with central obesity is associated with an imbalance of homeostatic mechanisms, leading to adipose tissue dysfunctionality characterized by altered secretion of adipokines. This condition is also associated with a special upregulation in the expression of pro-inflammatory adipokines[12] and increase in the generation of free radicals and other reactive species, leading to increased oxidative stress, production of cell adhesion molecules, and microcirculation dysfunction[13].

The prevalence of CAN is variable and is dependent on the definition and criteria used for the diagnosis. Toronto Consensus recommends using four cardiovascular reflex tests (CARTs) and frequency-domain tests as a sensitive and specific method in assessing the presence of CAN in patients with DM[14]. Furthermore, the American Academy of Neurology's summary of evidence-based guidelines for clinicians recommends that a combination of autonomic screening tests and composite autonomic scoring scales (CASS) should be considered to achieve the highest diagnostic accuracy of CAN[15]. These two diagnostic methods and scoring systems are commonly used in research and clinical practice.

CAN has a strong influence on various cardiovascular diseases and leads to severe morbidity and mortality in patients with DM[16, 17]. To our knowledge, only a few studies have demonstrated that adipocytokines are associated with autonomic dysfunctions in type 1 diabetes mellitus (T1DM) and T2DM $[2,18-20]$. To date, there is paucity of information that focuses on estimating the overall severity 
of CAN and investigates its relationship with the biomarkers for adipokines (leptin, chemerin, adiponectin, and vaspin), oxidative stress, and endothelial dysfunction in patients withT1DM and T2DM, respectively.

This aimed to identify adipocytokines strongly associated with the presence of CAN and investigate the severity of CAN in patients with T2DM using two CAN scoring systems. Our results may be beneficial for the development of therapeutic strategies for patients with DM and may help improve the quality of life of patients with T2DM and prediabetes.

\section{Patients And Methods}

\section{Study population}

A total of 107 patients ( $\geq 20$ years of age) who visited the outpatient diabetic clinic at Kaohsiung Chang Gung Memorial Hospital (CGMH) in Taiwan were included in this study: 90 subjects with type 2 diabetes and 17 subjects with prediabetes. Exclusion criteria were moderate-to-severe heart failure (NYHA class III and IV), presence of any type of arrhythmia that prevents analysis of heart rate variability (HRV), or pacemaker implantation. This study was approved by the Ethics Committee of Chang Gung Memorial Hospital Institutional Review Board (201800388B0C501 and 201901363B0).

\section{Baseline clinical and laboratory measurements}

All patients underwent complete neurological and physical examinations on enrollment and at the followup visit at the outpatient clinic. A detailed medical history regarding prior use of medications was obtained from the patients and their families through standardized questions. Demographic data, including age, sex, duration of diabetes (years), body mass index (BMI), systolic and diastolic blood pressure (SBP and DSP, respectively), waist circumference (WC) during autonomic function testing, underlying diseases (hypertension, coronary artery disease, ischemic stroke, and diabetic retinopathy [DR]), and laboratory parameters, were obtained at baseline. MetS was evaluated according to the updated National Cholesterol Education Program/Adult Treatment Panel III criteria[21]. A subject who had at least three of the following components was defined as having MetS: 1) central obesity: $W C \geq 90 \mathrm{~cm}$ for men and $\geq 80 \mathrm{~cm}$ for women; 2) hypertension: SBP $\geq 130 \mathrm{mmHg}$ or DBP $\geq 85 \mathrm{mmHg}$ or drug treatment for hypertension; 3) fasting blood glucose $\geq 100 \mathrm{mg} / \mathrm{dL}$ or diagnosed diabetes; 4) abnormal high-density lipoprotein (HDL) level: $\mathrm{HDL}$ cholesterol level $<40 \mathrm{mg} / \mathrm{dL}$ for men and $<50 \mathrm{mg} / \mathrm{dL}$ for women or drug treatment for low HDL cholesterol (HDL-C); and 5) abnormal triglyceride (TG) level: TG level $\geq$ $150 \mathrm{mg} / \mathrm{dL}$ or drug treatment for high TGs. The cut-off values for obesity, based on BMl, should be much lower in Taiwan than in Western countries. We determined that the optimal cut-off values for our study were BMls of 23.6 in men and 22.1 in women, and WCs of $80.5 \mathrm{~cm}$ in men and $71.5 \mathrm{~cm}$ in women may be more appropriate to define adult overweight or obesity in Taiwan[22].

\section{Measuring IR}

The homeostasis model assessment of insulin resistance (HOMA-IR index) was calculated by fasting glucose (in $\mathrm{mmol} / \mathrm{L}$ ) $\times$ fasting insulin (in $\mathrm{mU} / \mathrm{ml}$ )/22.5. In our study, we defined $\mathrm{IR}$ as $\geq 2$, based on the 
Taiwanese population[23]. Additionally, we used the TG/HDL ratio as a surrogate marker for IR[23].

\section{Assessment of albuminuria and glomerular filtration rate}

The estimated glomerular filtration rate (eGFR) in each patient was calculated using an equation for Chinese subjects, as previously described[24]. The normal rate of albumin excretion is less than $30 \mathrm{mg} /$ day; therefore, persistent albumin excretion between 30 and $300 \mathrm{mg} /$ day is classified as microalbuminuria and albumin excretion above $300 \mathrm{mg} /$ day is considered macroalbuminuria[25].

\section{Biochemical analysis}

Blood samples were obtained by antecubital vein puncture in a fasting, non-sedative state between 09:00 and 10:00 AM in the control and study groups to exclude the possible influence of circadian variations. All blood samples were collected into Vacutainer SST tubes (BD, Franklin Lakes, $\mathrm{NJ}$ ) and centrifuged at $3000 \mathrm{rpm}$ for 10 minutes; subsequently, serum samples were collected and stored at $-80^{\circ} \mathrm{C}$ in multiple aliquots, prior to biochemical measurement.

Serum levels of TGs, total cholesterol, HDL-C, low-density lipoprotein cholesterol, blood sugar, glycohemoglobin (HBA1c), and high-sensitive C-reactive protein (hs-CRP) were analyzed by the hospital's central laboratory.

\section{Biomarkers for oxidative stress}

We evaluated the oxidative stress condition in all subjects by measuring the serum thiobarbituric acidreactive substance (TBARS) and thiol levels. Serum TBARS levels were measured using a wellestablished method for detecting lipid peroxidation with a commercially available assay kit (Cayman Chemical, Ann Arbor, Michigan, USA). The Assay Kit was used according to the manufacturer's instructions, as previously described[26]. The values for the samples were calculated using a linear calibration curve, which was prepared using pure malondialdehyde-containing samples (range, 0$50 \mu \mathrm{mol} / \mathrm{L})$.

The ability of anti-oxidative defense in response to increased oxidative damage was evaluated by measuring the serum levels of total reduced thiols because serum thiols are physiologic free radical scavengers. Serum total protein thiols were estimated by directly reacting thiols with 5,5 -dithiobis 2 nitrobenzoic acid to form 5-thio-2-nitrobenzoic acid (TNB). The number of thiols in the sample was calculated based on absorbance, which was determined using the extinction coefficient of TNB (A412 = $13,600 / \mathrm{M} / \mathrm{cm})$.

\section{ELISA Analysis for biomarkers of endothelial dysfunction and adipokines}

Serum sICAM-1 and sVCAM-1 levels were assessed using commercially available ELISA kits (R \& D Systems, Minneapolis, MN, USA), as previously described[27]. Leptin and adiponectin levels were evaluated in the quality-controlled central laboratory of the CGMH. Serum chemerin levels were assessed 
by duplicated determination with a commercially available ELISA kit (R\&D Systems, Inc., Minneapolis, MN, USA), according to the manufacturer's instructions, and the mean minimum detectable dose was $4.13 \mathrm{pg} / \mathrm{mL}$.

\section{Assessment and scoring of cardiovascular autonomic functions}

All subjects underwent a standardized evaluation of cardiovascular autonomic function, as described by Low[28]. The test battery comprised the heart rate response to deep breathing (HR_DB), Valsalva ratio (VR), and 5-minute head-up tilt (HUT) tests. Orthostatic SBP change was defined as the difference between minimum SBP during HUT and baseline SBP. The detailed methodology for computing HR_DB and VR were based on a previous study[28]. The severity of CAN was assessed using the cardiovagal and adrenergic sub-scores of the CASS[29]. The CASS had a scale from 0 to 7 points in this study, and patients with a CASS score $\geq 2$ were defined as having CAN (CASS-based)[30].

In addition, CARTs are considered gold-standard measures of autonomic function in patients with DM[14]. Parameters, which were computed as Ewing's methods, included heart rate responses to deep breathing (E:I ratio), to standing (30:15 ratio), and to the Valsalva maneuver and blood pressure responses to standing[31], were often used by diabetologists. These autonomic parameters were also obtained, and the severity of CAN was quantitated by summation of points obtained from each of the four tests (CARTs score), where each test was given a point of 0 or 1 , if it yielded normal or abnormal values. The CARTs provided a score of 0 to 4 points in this study. CAN was defined with the presence of at least two abnormal test results, i.e., CARTs score $\geq 2$ (CARTs-based)[14].

\section{Assessment of baroreflex sensitivity}

A 5-minute resting recording of an EKG was obtained before the 5-minute HUT test. Non-invasive estimates of baroreflex sensitivity (BRS) were computed with the recording, using the sequence method of the Nevrokard BRS analysis program (Nevrokard, Slovenia). The following setting was used in computing BRS: 1) RR interval (RRI) variation greater than $5 \mathrm{~ms} ; 2$ ) SBP changes $>1 \mathrm{mmHg}$; 3 ) sequences longer than 3 beats; and 4) correlation coefficient $>0.85$. Patient with both bradycardic (increase in SBP that caused an increase in RRI) and tachycardic sequences (decrease in SBP that caused a decrease in $\mathrm{RRI}$ ) that fulfilled the criteria, were used in the analysis. Fluctuations of RRI and SBP were synchronous for some subjects, while in other subjects a time-lag between these two fluctuations was observed. Therefore, BRS was calculated using synchronous mode as well as shift mode from 1 to 6 heart beat shifts for each subject[32]. The mode with the largest number of slopes was selected, and the averaged slope of regression lines was used as the measure of BRS.

\section{Parameters of HRV}

The standard deviation of all normal R-R intervals (SDNN) was calculated using the 5-minute resting EKG recording as the time domain parameter for HRV. In addition, power spectral density analysis of HRV was also performed to obtain the parameters in the frequency domain. Three main spectral components were 
distinguished in a spectrum calculated from the 5-minute recording: high frequency $(\mathrm{HF}, 0.15-0.4 \mathrm{~Hz})$, low frequency $(L F, 0.04-0.15 \mathrm{~Hz})$, and very low frequency $(0-0.04 \mathrm{~Hz})$. The components of $L F$ and HF were computed both in absolute values of power $\left(\mathrm{ms}^{2}\right)$ and in normalized unit (n.u.). The LF/HF ratio, regarded as an index of sympatho-vagal balance, was also calculated[33]. The above computing process was performed using Kubios HRV Standard version 3.2 (Kubios Oy, Finland)

\section{Statistical analysis}

Data are expressed as means \pm standard derivations or medians (interquartile ranges). Categorical variables were compared using Chi-square or Fisher's exact tests. Continuous variables that were not normally distributed by Kolmogorov-Smirnov test were logarithmically transformed to improve normality and compared. Five separate statistical analyses were performed. First, patients were stratified into two groups according to the presence or absence of CAN by the CASS-based method. Second, the risk factors for the presence of CAN, including sex and baseline characteristics, underlying diseases, and parameters of laboratory testing, were analyzed using stepwise logistic regression. Third, receiver operating characteristic (ROC) curves were generated for significant adipocytokines levels in the presence of CAN by both CASS-based and CARTs-based CAN. The area under the ROC curves (AUC) was calculated for the presence of CAN. Fourth, correlation analysis was used to evaluate the relationship between the CASS and variables that included age, diabetes duration, BMI, WC, SPB, and DSP, and peripheral blood studies for vascular risks. We also used correlation analysis to evaluate the relationship between leptin and variables related to vascular risk factors. Finally, stepwise models of multiple linear regression analysis were used to evaluate the influence of independent variables on the mean CASS and CARTS scores. All statistical analyses were conducted using the SAS software package, version 9.1 (2002, SAS Statistical Institute, Cary, North Carolina).

\section{Results}

\section{General characteristics of the patients with diabetes and prediabetes}

A total of 107 patients were included in this study: 90 with diabetes and 17 with prediabetes. Patient characteristics and baseline underlying diseases at assessment are presented in Table 1. The mean diabetes duration in the diabetes group was $11.5 \pm 8.6$ years. The significant differences between the diabetes and prediabetes groups included BMI $(p=0.001)$, WC $(p=0.001)$, presence of chronic kidney diseases $(p=0.002)$, and proteinuria $(p=0.001)$. 
Table 1

Baseline characteristics of patients with Type 2 diabetes and prediabetes

Prediabetes $(n=17) \quad$ Diabetes $(n=90)$

P-value

Characteristics

Age (year)

$63.3 \pm 12.8$

$67.3 \pm 8.1$

0.21

Sex (male/female)

$11 / 6$

$50 / 40$

0.49

Diabetes duration (year)

$11.5 \pm 8.6$

Body mass index

$24.3 \pm 2.5$

$27.1 \pm 4.6$

0.001 *

Waist circumference $(\mathrm{cm})$

$85.3 \pm 7.5$

$94.6 \pm 11.7$

0.001 *

$S B P(m m H g)$

$131.6 \pm 14.8$

$137.8 \pm 17.9$

0.07

$D B P(m m H g)$

$76.9 \pm 8.2$

$76.5 \pm 12.5$

0.90

Baseline underlying disease

Hypertension (\%)

11

68

0.38

Coronary heart disease (\%)

2

Cerebrovascular events (\%)

4

Hyperlipidemia (\%)

14

Chronic kidney diseases (\%)

2

Retinopathy, $n$ (\%)

5

Proteinuria, $n(\%)$

0

11

1.0

43

0.30

Type of diabetes treatment

OHA only (\%)

0

$74(82.2 \%)$

Insulin only (\%)

0

$4(4.4 \%)$

Insulin + OHA (\%)

0

$7(7.8 \%)$

Diet control without medication (\%)

$17(100 \%)$

$5(5.6 \%)$

Other concomitant medications

ACE inhibitor or ARB (\%)

11 (64.7\%)

$65(72.2 \%)$

Beta-blocker (\%)

$3(17.6 \%)$

$28(31.1 \%)$

Calcium channel blocker (\%)

$4(23.5 \%)$

$45(50 \%)$

Diuretics (\%)

$3(17.6 \%)$

$18(20 \%)$ 


\begin{tabular}{|c|c|c|c|}
\hline & Prediabetes $(n=17)$ & Diabetes $(n=90)$ & $P$-value \\
\hline Antiplatelet medications (\%) & $4(23.5 \%)$ & $26(28.9 \%)$ & \\
\hline Statins (\%) & $13(76.4 \%)$ & 70 (77.8\%) & \\
\hline \multicolumn{4}{|c|}{$\begin{array}{l}\text { * indicates that } p \text { value }<0.05 . * * \text { indicates that } p \text { value }<0.0001 \text {. } \\
\text { Abbreviations: } n, \text { number of cases; IQR = interquartile range, SBP, systolic blood pressure; } D B P, \\
\text { diastolic blood pressure; OHA, oral hypoglycemic agent; } A C E, \text { angiotensin-converting enzyme; } A R B \text {, } \\
\text { angiotensin II receptor blocker }\end{array}$} \\
\hline
\end{tabular}


Table 2

Risk factors predictive of cardiovascular autonomic neuropathy in patients with Type 2 diabetes and prediabetes

\begin{tabular}{|c|c|c|c|c|c|}
\hline & $\begin{array}{l}\text { No CAN } \\
(n=59)\end{array}$ & $\begin{array}{l}\text { CAN } \\
(n=48)\end{array}$ & $\begin{array}{l}\text { Crude P- } \\
\text { value }^{\Phi}\end{array}$ & $\begin{array}{l}\text { Adjusted } \\
\text { OR ( } 95 \% \mathrm{Cl})\end{array}$ & $\begin{array}{l}\text { Adjusted } \\
P \text {-value }\end{array}$ \\
\hline \multicolumn{6}{|l|}{ Characteristics } \\
\hline Age (year) & $\begin{array}{l}67.5 \pm \\
9.7\end{array}$ & $66.1 \pm 8.3$ & 0.41 & & \\
\hline Sex (male/female) & $36 / 23$ & $25 / 23$ & 0.35 & & \\
\hline Diabetes duration (year) & $7.0 \pm 6.5$ & $12.8 \pm 9.6$ & $0.001^{* *}$ & $\begin{array}{l}1.08(1.02- \\
1.15)\end{array}$ & 0.011 \\
\hline Body mass index & $\begin{array}{l}26.2 \pm \\
4.2\end{array}$ & $27.1 \pm 4.8$ & 0.3 & & \\
\hline Waist & $\begin{array}{l}91.5 \pm \\
10.8\end{array}$ & $\begin{array}{l}95.8 \pm \\
12.3\end{array}$ & 0.07 & & \\
\hline$S B P(m m H g)$ & $\begin{array}{l}134.0 \pm \\
17.6\end{array}$ & $\begin{array}{l}140.3 \pm \\
17.0\end{array}$ & 0.07 & & \\
\hline$D B P(m m H g)$ & $\begin{array}{l}75.5 \pm \\
71.3\end{array}$ & $\begin{array}{l}77.8 \pm \\
12.5\end{array}$ & 0.32 & & \\
\hline Retinopathy, n (\%) & $\begin{array}{l}32 \\
(54 \%)\end{array}$ & $26(54 \%)$ & 0.99 & & \\
\hline Proteinuria, $n(\%)$ & $\begin{array}{l}13 \\
(22 \%)\end{array}$ & $24(50 \%)$ & $0.002^{* *}$ & & \\
\hline \multicolumn{6}{|l|}{ Laboratory test findings } \\
\hline Total cholesterol(mmol/L) & $\begin{array}{l}158.7 \pm \\
34.4\end{array}$ & $\begin{array}{l}158.3 \pm \\
32.7\end{array}$ & 0.96 & & \\
\hline Triglyceride(mmol/L) & $\begin{array}{l}129.1 \pm \\
71.8\end{array}$ & $\begin{array}{l}147.7 \pm \\
78.3\end{array}$ & 0.21 & & \\
\hline$H D L-C(m m o l / L)$ & $\begin{array}{l}48.8 \pm \\
14.0\end{array}$ & $44.3 \pm 8.9$ & 0.06 & & \\
\hline$L D L-C(\mathrm{mmol} L)$ & $\begin{array}{l}85.5 \pm \\
22.8\end{array}$ & $\begin{array}{l}87.7 \pm \\
29.8\end{array}$ & 0.69 & & \\
\hline $\begin{array}{l}H b A 1 c(\%) 6.9 \pm 0.97 .4 \pm 0.97 .3 \\
\pm 1.17 .4 \pm 1.30 .171\end{array}$ & $6.7 \pm 0.8$ & $7.1 \pm 0.8$ & $0.02^{*}$ & & \\
\hline Glucose Ac & $\begin{array}{l}133.1 \pm \\
31.4\end{array}$ & $\begin{array}{l}132.3 \pm \\
31.0\end{array}$ & 0.91 & & \\
\hline eGFR & $\begin{array}{l}59.4 \pm \\
30.2\end{array}$ & $\begin{array}{l}51.0 \pm \\
24.5\end{array}$ & 0.14 & & \\
\hline
\end{tabular}




\begin{tabular}{|c|c|c|c|c|c|}
\hline & $\begin{array}{l}\text { No CAN } \\
(n=59)\end{array}$ & $\begin{array}{l}\text { CAN } \\
(n=48)\end{array}$ & $\begin{array}{l}\text { Crude } P \text { - } \\
\text { value }{ }^{\Phi}\end{array}$ & $\begin{array}{l}\text { Adjusted } \\
\text { OR }(95 \% \mathrm{Cl})\end{array}$ & $\begin{array}{l}\text { Adjusted } \\
\text { P-value }\end{array}$ \\
\hline UACR & $\begin{array}{l}9.2(5.9 \\
21.5)\end{array}$ & $\begin{array}{l}39.9(8.5 \\
207.5)\end{array}$ & $0.004^{* *}$ & & \\
\hline$H s-C R P$ & $1.5 \pm 1.2$ & $2.2 \pm 1.8$ & 0.21 & & \\
\hline \multicolumn{6}{|l|}{$\begin{array}{l}\text { Biomarkers for Insulin } \\
\text { resistance }\end{array}$} \\
\hline HOMA-IR & $3.1 \pm 2.6$ & $3.7 \pm 2.8$ & 3.43 & & \\
\hline$T G / H D L$ ratio & $3.0 \pm 2.0$ & $3.6 \pm 2.2$ & 1.52 & & \\
\hline \multicolumn{6}{|c|}{ Biomarkers of adipocytokine } \\
\hline Leptin, $n g / m L$ & $\begin{array}{l}7.5(4.3 \\
15.7)\end{array}$ & $\begin{array}{l}15.1(9.9 \\
31.1)^{(9.9}\end{array}$ & $0.003^{* *}$ & $\begin{array}{l}1.03(1- \\
1.06)\end{array}$ & 0.023 \\
\hline Adiponectin, $\mathrm{ng} / \mathrm{mL}$ & $\begin{array}{l}11.6 \\
(6.7, \\
17.1)\end{array}$ & $\begin{array}{l}12.4(7.6 \\
24.3)\end{array}$ & 0.07 & & \\
\hline Chemerin, $n g / m L$ & $\begin{array}{l}85.9 \pm \\
23.6\end{array}$ & $\begin{array}{l}104.6 \pm \\
36.0\end{array}$ & $0.002^{\star *}$ & & \\
\hline Vaspin, $n g / m L$ & $\begin{array}{l}0.2(0.1 \\
0.3)\end{array}$ & $\begin{array}{l}0.2(0.1 \\
0.3)\end{array}$ & 0.82 & & \\
\hline \multicolumn{6}{|c|}{ Biomarkers of oxidative stress } \\
\hline TBARS, $\mu \mathrm{mol} / L$ & $\begin{array}{l}18.8 \pm \\
6.2\end{array}$ & $20.6 \pm 7.5$ & 0.19 & & \\
\hline Thiols, $\mu \mathrm{mol} / \mathrm{L}$ & $1.0 \pm 0.3$ & $0.9 \pm 0.4$ & $0.04^{*}$ & & \\
\hline \multicolumn{6}{|c|}{$\begin{array}{l}\text { Biomarkers of endothelial } \\
\text { dysfunction }\end{array}$} \\
\hline slCAM-1 (ng/ml) & $\begin{array}{l}203.9 \pm \\
71.0\end{array}$ & $\begin{array}{l}212.7 \pm \\
76.7\end{array}$ & 0.54 & & \\
\hline sVCAM-1 (ng/ml) & $\begin{array}{l}740.4 \pm \\
226.5\end{array}$ & $\begin{array}{l}818.3 \pm \\
260.1\end{array}$ & 0.1 & & \\
\hline \multicolumn{6}{|c|}{$\begin{array}{l}\text { Abbreviations: IQR = interquartile range, } C A N \text { : cardiac autonomic neuropathy; } n \text {, number of cases; SBP, } \\
\text { systolic blood pressure; DBP, diastolic blood pressure; } H D L-C \text {, high-density lipoprotein cholesterol; } L D L- \\
\text { C, low-density lipoprotein cholesterol; HbA1C, glycohemoglobin; HOMA-IR, Homeostasis model } \\
\text { assessment of insulin resistance; } T G / H D L \text {, Triglyceride/ LDL-C; }\end{array}$} \\
\hline
\end{tabular}




\section{Risk factors predictive of CAN in patients with type 2 diabetes and prediabetes}

Patients with CAN had higher diabetes duration $(P=0.001)$, higher HbA1c $(\%)(P=0.02)$, UACR $(P=$ $0.004)$, leptin $(P=0.003)$, and chemerin level $(P=0.002)$, and a higher prevalence of proteinuria $(P=$ $0.002)$ and lower thiol levels $(P=0.04)$. Significant variables used in the stepwise logistic regression model included diabetes duration, HbA1c (\%), UACR, leptin, chemerin and thiols level, and presence of proteinuria. After analysis of all the variables, only diabetes duration $(P=0.011, O R=1.08,95 \% \mathrm{Cl}=1.02-$ $1.15)$ and leptin level $(P=0.023, O R=1.03,95 \% C l=1-1.06)$ were independently associated with the presence of CAN.

\section{Parameters of cardiovascular autonomic study between patients with and without CAN}

Patients with CAN had higher CASS and CARTs score values, and lower levels of parasympathetic parameters, which include HR_DB (beats/min), VR, E:I ratio, 30:15 ratio, and sympathetic parameters, such as blood pressure responses to standing. Those with CAN had lower BRS values than those without CAN. Additional HRV parameters, including SDNN, total spectral power of LF and HF, LF (n.u.), HF (n.u.), and LF/HF ratio in patients with and without CAN are listed in Table 3. 
Table 3

Baseline cardiovascular autonomic study with Type 2 diabetes and prediabetes

\begin{tabular}{|c|c|c|c|}
\hline & No CAN $(n=59)$ & $\operatorname{CAN}(n=48)$ & $P$-value ${ }^{\Phi}$ \\
\hline$C A S S^{\delta}$ & $0.5 \pm 0.4$ & $3.2 \pm 1.1$ & $<0.0001^{* * *}$ \\
\hline$H R \_D B$ (beats/min) & $11.1 \pm 7.0$ & $5.5 \pm 3.9$ & $<0.0001^{* * *}$ \\
\hline Valsalva ratio & $1.4 \pm 0.2$ & $1.2 \pm 0.1$ & $<0.0001^{* * *}$ \\
\hline Delta SBP & $-3.0(-10.0,2.0)$ & $-8(-17.0,-2.0)$ & $0.004^{* *}$ \\
\hline CARTs score ${ }^{\beta}$ & $0.6 \pm 0.5$ & $2.4 \pm 0.7$ & $<0.0001^{* * *}$ \\
\hline E:I ratio ${ }^{\beta}$ & $1.2 \pm 0.1$ & $1.1 \pm 0.04$ & $<0.0001^{* * *}$ \\
\hline 30/15 ratio $^{\beta}$ & $1.1 \pm 0.1$ & $1.0 \pm 0.03$ & $<0.0001^{* * *}$ \\
\hline \multicolumn{4}{|l|}{ Time domain } \\
\hline$S D N N(m s)$ & $28.4 \pm 19.8$ & $18.2 \pm 8.0$ & $0.001^{* *}$ \\
\hline \multicolumn{4}{|l|}{ Frequency domain } \\
\hline$L F\left(\right.$ power density, $\left.m s^{2}\right)$ & $87.6(34.3,174.6)$ & $30.7(13.1,87.6)$ & $0.001^{* *}$ \\
\hline$L F$ (n. u.) & $26.2 \pm 4.2$ & $27.1 \pm 4.8$ & 0.145 \\
\hline HF (power density, $\left.m s^{2}\right)$ & $50.9(17.1,179.5)$ & $35.4(15.2,80.3)$ & 0.147 \\
\hline$H F$ (n. u.) & $53.8 \pm 21.9$ & $47.4 \pm 22.2$ & 0.145 \\
\hline$L F / H F$ ratio & $1.6(0.6,2.8)$ & $0.9(0.4,1.9)$ & 0.204 \\
\hline$B R S(m s / m m H g)$ & $6.8 \pm 4.4$ & $3.8 \pm 1.9$ & $<0.0001^{* * *}$ \\
\hline \multicolumn{4}{|c|}{$\begin{array}{l}\text { Data are presented as means } \pm \text { standard deviations or median }(I Q R) n(\%) . *=P<0.05 ; * \star P<0.01 \text {; } \\
\star \star \star *=P<0.001\end{array}$} \\
\hline \multicolumn{4}{|c|}{$\begin{array}{l}\text { Abbreviations: } n, \text { number of cases; } I Q R=\text { interquartile range, CASS: Composite Autonomic Scoring } \\
\text { Scale; } H R \text { DB, heart rate response to deep breathing; CAN, cardiac autonomic neuropathy; Delta SBP: } \\
\text { The change between minimum systolic blood pressure during head-up tilt and baseline systolic blood } \\
\text { pressure; CARTs, cardiac autonomic reflex tests; LF, low frequency; HF, high frequency; } n \text {.u., } \\
\text { normalized unit; BRS, baroreflex sensitivity }\end{array}$} \\
\hline \multicolumn{4}{|c|}{$\begin{array}{l}\Phi=\text { independent } t \text { - test, two-side, data were logarithmically transformed to improve normality; } \delta= \\
\text { Except CARTs score, E:I ratio, and } 30 / 15 \text { ratio, CAN defined by CASS-based method in the other } \\
\text { parameters, } \beta=\text { CAN defined by CARTs method }\end{array}$} \\
\hline
\end{tabular}

\section{Sensitivity, specificity, and area under the curve for significant adipocytokines levels in predicting}




\section{cardiovascular autonomic neuropathy, using ROC curve analysis}

The diagnostic accuracy for the presence of CAN in CASS-based CAN was as follows: leptin $(P<0.0001)$, chemerin $(P=0.002)$, adiponectin $(P=0.18)$, and vaspin $(P=0.9)$. The area under the ROC curve for leptin and chemerin levels in the presence of CAN were $0.71(95 \%$ confidence interval $[\mathrm{Cl}]=0.605-0.805)$ and $0.68(95 \% \mathrm{Cl}=0.578-0.784)$, respectively. The diagnostic accuracy for the presence of CAN in CARTsbased CAN was as follows: leptin ( $P=0.002)$, chemerin $(P=0.03)$, adiponectin $(P=0.82)$, and vaspin $(P=$ 0.62). The area under the ROC curve for leptin and chemerin levels in the presence of CAN were 0.69 (95\% $\mathrm{Cl}=0.581-0.793)$ and $0.63(95 \% \mathrm{Cl}=0.517-0.745)$, respectively.

\section{Correlation analysis between leptin levels and parameters of biomarkers and cardiovascular autonomic functions}

Correlation analysis parameters that are used to test the relationship between leptin level and parameters of biomarkers and cardiovascular autonomic functions are listed in Table 4. The significant statistical results (correlation coefficient, $P$-value) between leptin level and parameters of biomarkers were as follows: BMI $(r=0.588, P<0.0001)$, WC $(r=0.504, P<0.0001)$, UACR $(r=0.351, P=0.001)$, hs-CRP $(r=$ $0.291, P=0.006)$, TBARS $(r=0.259, P=0.008)$, thiols $(r=-0.25, P=0.011)$, TG/HDL-C ratio $(r=0.346, P<$ $0.0001)$, and HOMA-IR $(r=0.304, P=0.008)$. Further, significant statistical results (correlation coefficient, $P$-value) between leptin levels and parameters of cardiovascular autonomic functions were as follows: HR_DB $(r=-0.30, P=0.002)$, Valsalva ratio $(r=-0.31, P=0.002), E: I$ ratio $(r=-0.26, P=0.008), 30 / 15$ ratio $(r=-0.18, P=0.07), B R S(r=-0.26, P=0.008), S D N N(r=-0.18, P=0.08), L F(r=-0.24, P=0.016), H F(r=0.24, P$ $=0.016)$, and $L F / H F$ ratio $(r=-0.22, P=0.032)$. 
Table 4

Correlation analysis between leptin levels and parameters of biomarkers and cardiovascular autonomic functions

\begin{tabular}{|c|c|c|c|c|c|}
\hline \multirow[t]{2}{*}{ Variables } & \multicolumn{2}{|c|}{ Leptin, ng/mL } & \multirow[t]{2}{*}{ Variables } & \multicolumn{2}{|c|}{ Leptin, ng/mL } \\
\hline & $r$ & $P$ value & & $r$ & $P$ value \\
\hline Age (year) & -0.10 & 0.33 & $H R \_D B$ & -0.30 & $0.002^{* *}$ \\
\hline Diabetes duration (year) & -0.023 & 0.821 & Valsalva ratio & -0.31 & $0.002^{* *}$ \\
\hline Body mass index & 0.588 & $<0.0001^{* *}$ & Delta SBP & 0.031 & 0.80 \\
\hline Waist circumference $(\mathrm{cm})$ & 0.504 & $<0.0001^{* *}$ & E:I ratio & -0.26 & $0.008^{* *}$ \\
\hline$S B P(m m H g)$ & 0.007 & 0.946 & $30 / 15$ ratio & -0.18 & 0.07 \\
\hline$D B P(m m H g)$ & -0.053 & 0.597 & $B R S$ & -0.26 & $0.008^{* *}$ \\
\hline$H b A 1 c(\%)$ & 0.175 & 0.076 & SDNN & -0.18 & 0.08 \\
\hline eGFR & -0.175 & 0.09 & $L F$, n.u. & -0.24 & $0.016^{*}$ \\
\hline UACR & 0.351 & $0.001^{* *}$ & $H F, n . u$ & 0.24 & $0.016^{*}$ \\
\hline$h s-C R P$ & 0.291 & $0.006^{* *}$ & $L F / H F$ ratio & -0.22 & $0.032^{*}$ \\
\hline TBARS, $\mu \mathrm{mol} / \mathrm{L}$ & 0.259 & $0.008^{* *}$ & & & \\
\hline Thiols, $\mu \mathrm{mol} / \mathrm{L}$ & -0.25 & $0.011^{*}$ & & & \\
\hline slCAM-1 (ng/ml) & 0.122 & 0.216 & & & \\
\hline sVCAM-1 (ng/ml) & 0.109 & 0.271 & & & \\
\hline Triglyceride/HDL-C ratio & 0.346 & $<0.0001$ & & & \\
\hline HOMA-IR & 0.304 & 0.008 & & & \\
\hline \multicolumn{6}{|c|}{$\begin{array}{l}\text { Abbreviations: n, number of cases; SBP, systolic blood pressure; DBP, diastolic blood pressure; HDL-C, } \\
\text { high-density lipoprotein cholesterol; } L D L-C \text {, low-density lipoprotein cholesterol; HbA1C, } \\
\text { glycohemoglobin; HOMA-IR :homeostasis model assessment of IR; HR_DB, heart rate response to } \\
\text { deep breathing; Delta SBP: The change between minimum systolic blood pressure during head-up tilt } \\
\text { and baseline systolic blood pressure, BRS: baroreflex sensitivity; } n . u \text {. =normalized unit; } L F, \text { low } \\
\text { frequency; HF, high frequency }\end{array}$} \\
\hline
\end{tabular}

\section{Effect of adipocytokines, MetS, and vascular risk factors on composite autonomic scoring scale and cardiac autonomic reflex test scores}


Correlation analysis parameters that are used to test the influence of adipocytokine, MetS, and vascular risk factors on CASS and CARTs scores are listed in Table 5. The significant statistical results (correlation coefficient, P-value) for CASS were as follows: diabetes duration (year) $(r=0.36, P<0.0001)$, WC $(r=0.24$, $P=0.02)$, eGFR $(r=-0.26, P=0.01)$, UACR $(r=0.36, P<0.0001)$, HbA1c $(\%)(r=0.38, P<0.0001)$, leptin $(\mathrm{ng} / \mathrm{mL})(r=0.36, P<0.0001)$, chemerin $(\mathrm{ng} / \mathrm{mL})(r=0.45, P<0.0001)$, TBARS $(\mu \mathrm{mol} / \mathrm{L})(r=0.26, P=$ $0.007)$, thiols $(\mu \mathrm{mol} / \mathrm{L})(r=-0.23, P=0.02)$, and $\operatorname{sVCAM}-1(\mathrm{ng} / \mathrm{ml})(r=0.24, P=0.01)$. The significant statistical results (correlation coefficient, P-value) on the CARTs score were as follows: age (year) $(r=-0.281, P=0.004)$, diabetes duration (year) $(r=0.247, P=0.015)$, leptin $(n g / m L)(r=0.356, P<0.0001)$, and TBARS $(\mu \mathrm{mol} / \mathrm{L})(r=0.188, P=0.011)$.

Table 5

Correlation analysis of composite autonomic scoring scale and cardiac autonomic reflex tests in patients with type 2 diabetes and pre-diabetes

\begin{tabular}{|c|c|c|c|c|}
\hline \multirow[t]{2}{*}{ Variables } & \multicolumn{2}{|l|}{ CASS } & \multicolumn{2}{|c|}{ CARTs score } \\
\hline & $r$ & $P$ value & $r$ & $P$ value \\
\hline Age (year) & -0.10 & 0.33 & -0.281 & $0.004^{*}$ \\
\hline Diabetes duration (year) & 0.36 & $<0.0001^{* *}$ & 0.247 & $0.015^{\star}$ \\
\hline Body mass index & 0.16 & 0.1 & 0.109 & 0.284 \\
\hline Waist circumference $(\mathrm{cm})$ & 0.24 & $0.02^{*}$ & 0.138 & 0.19 \\
\hline$S B P(m m H g)$ & 0.12 & 0.22 & 0.026 & 0.8 \\
\hline$D B P(m m H g)$ & -0.01 & 0.89 & -0.031 & 0.76 \\
\hline eGFR & -0.26 & $0.01^{*}$ & -0.143 & 0.161 \\
\hline$U A C R$ & 0.36 & $<0.0001^{* *}$ & 0.198 & 0.064 \\
\hline$H b A 1 c(\%)$ & 0.38 & $<0.0001^{* *}$ & 0.176 & 0.081 \\
\hline Leptin, $n g / m L$ & 0.36 & $<0.0001^{* *}$ & 0.356 & $0.001^{* *}$ \\
\hline Chemerin, $n g / m L$ & 0.45 & $<0.0001^{* *}$ & 0.254 & 0.217 \\
\hline TBARS, $\mu \mathrm{mol} / \mathrm{L}$ & 0.26 & $0.007^{*}$ & 0.188 & $0.011^{*}$ \\
\hline Thiols, $\mu \mathrm{mol} / \mathrm{L}$ & -0.23 & $0.02^{*}$ & -0.256 & 0.063 \\
\hline slCAM-1 (ng/ml) & 0.01 & 0.93 & -0.034 & 0.739 \\
\hline sVCAM-1 (ng/ml) & 0.24 & $0.01^{*}$ & 0.084 & 0.411 \\
\hline \multicolumn{5}{|c|}{$r:$ correlation coefficient. * indicates that $p$ value $<0.05 .{ }^{* *}$ indicates that $p$ value $<0.0001$} \\
\hline \multicolumn{5}{|c|}{$\begin{array}{l}\text { Abbreviations: } n, \text { number of cases; CASS, Composite Autonomic Scoring Scale; CARTs: Cardiac } \\
\text { autonomic reflex tests; SBP, systolic blood pressure; DBP, diastolic blood pressure; HDL-C, high-density } \\
\text { lipoprotein cholesterol; } L D L-C \text {, low-density lipoprotein cholesterol; HbA1C, glycohemoglobin; }\end{array}$} \\
\hline
\end{tabular}




\section{Effects of the variables on composite autonomic scoring scale and cardiac autonomic reflex tests}

Effects of variables on the CASS and CARTs scores in patients with T2DM based on the correlation analysis are listed in Table 6. Regarding the correlation analysis, only significant variables were enrolled into stepwise linear regressions models. The results from model analysis (Table 6) revealed that only diabetes duration, UACR, and leptin level were significantly associated with the CASS and only age, diabetes duration, and leptin level were significantly associated with the CARTs score. 
Table 6

Effects of the variables on composite autonomic scoring scale and cardiac autonomic reflex tests according to correlation analysis

\begin{tabular}{|c|c|c|c|c|c|c|c|}
\hline \multirow[t]{2}{*}{ variables } & \multicolumn{3}{|l|}{ CASS $^{a}$} & \multirow[t]{2}{*}{ variables } & \multicolumn{3}{|c|}{ CARTs score ${ }^{\beta}$} \\
\hline & $\begin{array}{l}\text { Model } \\
T^{r}\end{array}$ & $\begin{array}{l}\text { Model } \\
2^{V}\end{array}$ & $\begin{array}{l}\text { Model } \\
3^{V}\end{array}$ & & $\begin{array}{l}\text { Model } \\
T^{r}\end{array}$ & $\begin{array}{l}\text { Model } \\
2^{r}\end{array}$ & $\begin{array}{l}\text { Model } \\
3^{V}\end{array}$ \\
\hline $\begin{array}{l}\text { Diabetes } \\
\text { duration }\end{array}$ & $0.268^{*}$ & $0.307^{* *}$ & $0.058 * *$ & $\begin{array}{l}\text { Diabetes } \\
\text { duration }\end{array}$ & $0.268^{* *}$ & $0.028 * *$ & $0.028 * *$ \\
\hline Leptin & $0.285^{*}$ & $0.014^{*}$ & $0.016^{* *}$ & Age & $-0.036 * *$ & $-0.032 * *$ & $-0.035^{\star *}$ \\
\hline$U A C R$ & $0.001^{* *}$ & $0.001^{*}$ & $0.001^{*}$ & Leptin & $0.243^{*}$ & $0.246^{*}$ & $0.008^{*}$ \\
\hline eGFR & -0.03 & -0.007 & 0.093 & TBARS & $0.207^{*}$ & 0.173 & 0.115 \\
\hline Chemerin, & 0.147 & -0.013 & -0.049 & sVCAM-1 & 0.152 & 0.11 & 0.076 \\
\hline Waist & 0.199 & 0.067 & 0.024 & & & & \\
\hline$H b A 1 c(\%)$ & 0.234 & 0.203 & 0.128 & & & & \\
\hline TBARS & 0.102 & 0.061 & 0.05 & & & & \\
\hline Thiols & -0.101 & -0.039 & 0.007 & & & & \\
\hline sVCAM-1 & $0.033^{*}$ & 0.052 & 0.041 & & & & \\
\hline \multicolumn{8}{|c|}{$\begin{array}{l}y=\text { Regression coefficient for each individual variable. Abbreviations: HbA1c, glycohemoglobin; CASS, } \\
\text { Composite Autonomic Scoring Scale; CARTs: Cardiac autonomic reflex tests; }{ }^{*} \text { indicates that } p \text { value }< \\
0.05 .{ }^{* *} \text { indicates that } p \text { value }<0.01\end{array}$} \\
\hline \multicolumn{8}{|c|}{ Model 1: Predictors:(constant), UACR; $r^{2}=0.15$} \\
\hline \multicolumn{8}{|c|}{ Model 2: Predictors:(constant), UACR, leptin; $r^{2}=0.22$} \\
\hline \multicolumn{8}{|c|}{ Model 3: Predictors:(constant), UACR, leptin and diabetes duration; $r^{2}=0.32$} \\
\hline \multicolumn{8}{|c|}{$\beta:$ Model summary } \\
\hline \multicolumn{8}{|c|}{ Model 1: Predictors: (constant), age; $r^{2}=0.13$} \\
\hline \multicolumn{8}{|c|}{ Model 2: Predictors:(constant), age, diabetes duration; $r^{2}=0.19$} \\
\hline Model 3: PI & tors:(cons & ant), age, & jabetes du & 1, leptin; $r^{2}=$ & & & \\
\hline
\end{tabular}

\section{Discussion}

\section{Major findings of our study}


To date, this is the first study to demonstrate that leptin level is not only strongly associated with the presence, but also with the degree of severity of CAN in patients with T2DM and prediabetes, assessed by both the CASS and CARTs scoring methods. All vascular risk factors, including blood pressure, blood glucose, and lipid profiles, were well-controlled, except for obesity, a modified risk factor, which was not corrected in our study. Our study also showed that leptin level is significantly correlated with BMI and WC. Thus, our results highlighted the relationship between leptin, obesity, and CAN.

\section{Pathogenesis of leptin and inflammation, oxidative stress, and endothelial dysfunction}

Visceral adipose tissue is a bioactive organ that secretes several adipokines, and is a source of proinflammatory and proatherogenic cytokines[34]. The rapid expansion of adipocyte size in obese individuals occurs in an uncoordinated manner, which leads to altered secretion of adipocytokines with a special upregulation in the expression of pro-inflammatory adipocytokines[12], as well as impaired angiogenesis, endothelial dysfunction, and microvascular complications[13].

Leptin and its receptors structurally resemble proinflammatory cytokines and their receptors[35]. In addition, proinflammatory mediators increase leptin secretion, and leptin levels increase acutely during inflammation[36]. Leptin may also increase oxidative stress via activation of the Rho and Rac family of small GTPase[37, 38]. Due to the proinflammatory and prooxidant properties of leptin, hyperleptinemia produces systemic endothelial dysfunction[37]. Our study results showed a positive association between leptin levels and inflammation (hs-CRP), increased oxidative stress (TBARs), and decreased antioxidative capacity (thiol). It also showed that leptin levels are positively associated with biomarkers of microvascular complications of diabetes (e.g., diabetic kidney diseases and CAN).

\section{Association among leptin, obesity, IR, and cardiac autonomic function in diabetes and pre-diabetes}

Leptin is a hormone predominantly synthesized by adipocytes and enterocytes in the small intestine that helps regulate energy balance and acts on cell receptors in the arcuate nucleus of the hypothalamus[6]. Although the regulation of fat stores is deemed to be the primary function of leptin, it also plays a role in other physiological processes. Leptin levels are generally elevated in obesity, and obese individuals are often leptin resistant, as seen in the failure of recombinant leptin to cause weight loss. Hyperleptinemia shunts excess free fatty acids ectopically to non-adipose tissue, and diverts fatty acids in those tissue to storage, rather than to oxidative consumption[39]. The lipotoxicity, ensuing from this ectopic accumulation of intracellular TGs, contributes to the dysfunction of these organs, and is a critical determinant of IR[40].

Although leptin levels are closely associated with adiposity and increased MetS components, leptin levels in diabetes and prediabetes is rather controversial[41, 42]. A recent study from Japan showed that plasma leptin level was comparable between diabetic and non-diabetic patients, despite the fact that BMI, visceral fat area, and subcutaneous fat area are significantly higher in patients with diabetes[18]. Another 
study in relatively lean rural Chinese adults found that plasma leptin levels were associated with IR and prediabetes, which were not totally explained by adiposity[43]. Our study results also showed that leptin levels are significantly correlated with both TG/HDL-C ratio and HOMA-IR index, the biomarkers of IR.

Leptin affects autonomic function by acting both centrally and peripherally. Leptin receptors in hypothalamic brain regions, implicated in cardiovascular control, may exert a stimulatory effect on sympathetic activation, which results in autonomic hyperactivity due to direct effects of neuropeptide systems, such as the melanocortin and corticotropin-releasing hormone[44]. Additionally, leptin receptors are abundantly present in the carotid body (CB)[45]. CBs contain glomus, which are polymodal chemoreceptors, also known as peripheral chemoreceptors that detect multiple chemical changes in oxygen, carbon dioxide, $\mathrm{PH}$, insulin, and blood sugar levels. A recent study showed that leptin increases the carotid sinus nerve activity[46], which transmits chemosensory input from the carotid bodies to the medullary centers (central chemoreceptors) in a mouse model. However, it is not yet clear why plasma leptin and autonomic function in patients with diabetes and prediabetes. Obesity triggers inflammation and oxidative stress, which activates the carotid bodies. The overactivation of the carotid bodies can contribute to increased sympathetic system activity and lead to hypertension and IR in T2DM[47].

\section{Risk factors associated with the severity of CAN}

The pathophysiological mechanism of CAN development is multifactorial, and several studies have reported the important role of cardiovascular risk factors[10,48-50]. However, in our study, degree of glycemic control and diabetes duration were the most common risk factors for both T1D and T2D. Our study results also demonstrate that diabetes duration is strongly associated with severity of CAN. CAN is a length-dependent pattern of disease, and parasympathetic activity can be damaged in the early phase of CAN with autonomic imbalance. As the disease progresses, sympathetic denervation occurs in the late stage of CAN[51]. Our results also showed that all parasympathetic parameters included in our study (e.g., HR_DB, VR, E:I ratio, 30:15 ratio, SDNN, HF power) were significantly lower in patients with CAN than in those without CAN; however, the difference between sympathetic parameters (LF power, orthostatic BP change) was not obvious between groups. This finding is compatible with that of previous reports[2, 18 , 19].

Regarding research on the relationship between leptin and CAN, one study from Japan showed that leptin is specifically associated with reduced HRV parameters in patients with DM compared with those without DM, with full adjustment of clinical parameters, which comprise quantitatively determined visceral adiposity[18]. Our results also showed that leptin level is negatively correlated with all parasympathetic parameters included in our study (e.g., HR_DB, VR, E:I ratio, 30/15 ratio, BRS, and SDNN). Another study from Korea showed a borderline significant positive correlation between leptin level and CAN score[19]. Although leptin is a sympathetic activator, this study from Korea showed that leptin levels were negatively correlated with the LF [19], and it is consistent with the result of our study. Such results are compatible with the natural course of CAN and are predominantly involved in the parasympathetic domain[51]. Autonomic dysfunction is seen early in the course of $\mathrm{DM}$, and it occurs alongside alterations of various inflammatory adipocytokines[2]. Therefore, it highlights the concept of autonomic imbalance in the 
pathogenesis of CAN. With the progression of CAN, cardiovagal impairment is followed by sympathetic impairment. Increased leptin level could be secondary to autonomic imbalance or obesity-related leptin resistance[2].

Another important issue is whether the medications for modifying vascular risk factors (e.g., antihypertensive, antihyperlipidemic, and antihyperglycemic agents) can decrease leptin levels. One systematic review for meta-analysis of randomized placebo-controlled trials (RCTs) focused on the impact of statin therapy on plasma leptin levels and showed that results are inconclusive[52]. Another meta-analysis of RCTs investigated the effects of pioglitazone on blood leptin levels in patients with T2DM and showed a significant difference, although relatively few RCTs were included, and a high level of statistical heterogeneity were found. Current studies have demonstrated that some antihypertensive medications might be more relevant than others in terms of reducing leptin levels in obesity. It seems that focus on pharmacologic suppression of the sympathetic nervous system, the renin-angiotensin system, and the blood pressure reduction effect is induced by weight loss. However, the results are also inconclusive[53]. Although our patients were well-controlled and almost all of them received medications to control the vascular risk factors, leptin levels remained significantly associated with the severity of CAN, according to both the CASS and CARTs score.

\section{Study Limitations}

This study has several limitations. First, visceral adipose tissue is a bioactive organ that secretes several adipokines (e.g., leptin) and proatherogenic cytokines, involved in cardiovascular events. A previous clinical study showed that visceral obesity revealed a more significant correlation with IR compared to central obesity, assessed by WC[54]. Other quantitative approaches to assess visceral obesity, such as dual bioelectrical impedance analysis for visceral fat measurement[55], should be considered for future studies. Second, current evidence supports that aerobic exercise, alone or combined with hypocaloric diet, improves symptoms of MetS, and also alters systemic levels of adipokines[56]. This is a cross-sectional prospective study. Clinical studies concluded that intensified multifactorial intervention (hyperglycemia, hypertension, dyslipidemia, and microalbuminuria) reduced the risk of CAN progression[57] in T2D and T1D[58]. Although we observed a close relationship between leptin level and severity of CAN in our observational study, it is unclear whether improvement of obesity could decrease leptin levels and simultaneously improve cardiovascular autonomic function.

\section{Conclusion}

Based on our results, leptin level is not only strongly associated with the presence, but also with the degree of severity of CAN in patients with T2D and prediabetes. As leptin level is considered a prognostic factor, a longitudinal study is needed to confirm that control of obesity can decrease leptin levels and can be effective in reducing $\mathrm{CAN}$ progression.

\section{Abbreviations}


T1DM and T2DM: type 1 and type 2 diabetes mellitus; CAN: cardiovascular autonomic neuropathy; CASS: composite autonomic scoring scale; CARTs: the cardiac autonomic reflex tests; TBARS: thiobarbituric acid-reactive substance; HDL-C: high-density lipoprotein cholesterol; HOMA-IR: homeostasis model assessment of insulin resistance; IR: insulin resistance; MetS: metabolic syndrome; HRV: heart rate variability; BMI: body mass index; SBP and DSP: systolic and diastolic blood pressure; WC: waist circumference; DR: diabetic retinopathy; TG: triglyceride; eGFR: estimated glomerular filtration rate; HBA1c: glycohemoglobin; hs-CRP: high-sensitive C-reactive protein; HR_DB: heart rate response to deep breathing; VR: Valsalva ratio; HUT : head-up tilt tests; BRS: baroreflex sensitivity; RRI: RR interval; SDNN: standard deviation of all normal R-R intervals; HF : high frequency; LF : low frequency; ROC: receiver operating characteristic; AUC: area under the ROC curves; CB: carotid body; RCTs : placebo-controlled trials;

\section{Declarations}

\section{Authors' contributions}

YRL participated in the design of the study and drafted the manuscript. WCC, CCH, NWT, BCC, and JFC participated in the sequence alignment and clinical evaluation of patients. $\mathrm{CCH}$ performed the statistical analysis. $\mathrm{CHL}$ and $\mathrm{CCH}$ conceived the idea for the study and participated in its design and coordination. They also helped drafting the manuscript. All authors read and approved the final manuscript.

\section{Author details}

${ }^{1}$ Department of Biological Science, National Sun Yat-Sen University, Kaohsiung, Taiwan;

Departments of ${ }^{2}$ Neurology, ${ }^{3}$ Internal Medicine, and ${ }^{4}$ Center for Shockwave Medicine and Tissue Engineering, Kaohsiung Chang Gung Memorial Hospital, Chang Gung University College of Medicine, Kaohsiung, Taiwan;

${ }^{5}$ Department of Neurology, Xiamen Chang Gung Memorial Hospital, Xiamen, Fujian, China

\section{Acknowledgements}

The authors thank all the subjects who participated in this study. This work was supported by grants from Chang Gung Memorial Hospital (Chang Gung Medical Research Project CMRPG8H0501).

\section{Competing interests}

The authors declare that they have no competing interests.

\section{Availability of data and materials}

The data from this study can be acquired from the corresponding author upon reasonable request. 


\section{Consent for publication}

Not applicable.

\section{Ethical approval and consent to participate}

This study conformed to the guidelines of the Declaration of Helsinki, and the study has been approved by the Institutional Review Board of Chang Gung Memorial Hospital (201800388B0C501 and 201901363B0).

\section{Funding}

This work was supported by grants from the Chang Gung Memorial Hospital (Chang Gung Medical Research Project CMRPG8H0501 and CMRPG8J1211).

\section{References}

1. Collaboration NCDRF. Worldwide trends in body-mass index, underweight, overweight, and obesity from 1975 to 2016: a pooled analysis of 2416 population-based measurement studies in 128.9 million children, adolescents, and adults. Lancet. 2017;390(10113):2627-42.

2. Lieb DC, Parson HK, Mamikunian G, Vinik Al: Cardiac autonomic imbalance in newly diagnosed and established diabetes is associated with markers of adipose tissue inflammation. Exp Diabetes Res. 2012;2012:878760.

3. Unamuno X, Gómez-Ambrosi J, Rodríguez A, Becerril S, Frühbeck G, Catalán V. Adipokine dysregulation and adipose tissue inflammation in human obesity. Eur J Clin Invest. 2018;48(9):e12997.

4. Rosen ED, Spiegelman BM. Adipocytes as regulators of energy balance and glucose homeostasis. Nature. 2006;444(7121):847-53.

5. Rodríguez A, Ezquerro S, Méndez-Giménez L, Becerril S, Frühbeck G. Revisiting the adipocyte: a model for integration of cytokine signaling in the regulation of energy metabolism. Am J Physiol Endocrinol Metab. 2015;309(8):E691-714.

6. Brennan AM, Mantzoros CS. Drug Insight: the role of leptin in human physiology and pathophysiology-emerging clinical applications. Nat Clin Pract Endocrinol Metab. 2006;2(6):31827.

7. Tilg H, Moschen AR. Role of adiponectin and PBEF/visfatin as regulators of inflammation: involvement in obesity-associated diseases. Clin Sci (Lond). 2008;114(4):275-88.

8. Goralski KB, McCarthy TC, Hanniman EA, Zabel BA, Butcher EC, Parlee SD, Muruganandan S, Sinal CJ. Chemerin, a novel adipokine that regulates adipogenesis and adipocyte metabolism. J Biol Chem. 2007;282(38):28175-88. 
9. Dimova R, Tankova T. The role of vaspin in the development of metabolic and glucose tolerance disorders and atherosclerosis. Biomed Res Int. 2015;2015:823481.

10. Valensi P, Paries J, Attali J, for Research FG. Cardiac autonomic neuropathy in diabetic patients: influence of diabetes duration, obesity, and microangiopathic complications-the French multicenter study. Metabolism. 2003;52(7):815-20.

11. Shin JA, Lee JH, Lim SY, Ha HS, Kwon HS, Park YM, Lee WC, Kang MI, Yim HW, Yoon KH. Metabolic syndrome as a predictor of type 2 diabetes, and its clinical interpretations and usefulness. J Diabetes Investig. 2013;4(4):334-43.

12. Ouchi N, Parker JL, Lugus JJ, Walsh K. Adipokines in inflammation and metabolic disease. Nat Rev Immunol. 2011;11(2):85.

13. Cao Y. Angiogenesis and vascular functions in modulation of obesity, adipose metabolism, and insulin sensitivity. Cell Metab. 2013;18(4):478-89.

14. Spallone V, Bellavere F, Scionti L, Maule S, Quadri R, Bax G, Melga P, Viviani G, Esposito K, Morganti R. Recommendations for the use of cardiovascular tests in diagnosing diabetic autonomic neuropathy. Nutr Metab Cardiovasc Dis. 2011;21(1):69-78.

15. England J, Gronseth G, Franklin G, Carter G, Kinsella L, Cohen J, Asbury A, Szigeti K, Lupski J, Latov N. Practice parameter: evaluation of distal symmetric polyneuropathy: role of autonomic testing, nerve biopsy, and skin biopsy (an evidence-based review): report of the American Academy of Neurology, American Association of Neuromuscular and Electrodiagnostic Medicine, and American Academy of Physical Medicine and Rehabilitation. Neurology. 2009;72(2):177-84.

16. Vinik Al, Maser RE, Mitchell BD, Freeman R. Diabetic autonomic neuropathy. Diabetes Care. 2003;26(5):1553-79.

17. Vinik Al, Ziegler D. Diabetic cardiovascular autonomic neuropathy. Circulation. 2007;115(3):387-97.

18. Kurajoh M, Koyama H, Kadoya M, Naka M, Miyoshi A, Kanzaki A, Kakutani-Hatayama M, Okazaki H, Shoji T, Moriwaki Y. Plasma leptin level is associated with cardiac autonomic dysfunction in patients with type 2 diabetes: HSCAA study. Cardiovasc Diabetol. 2015;14(1):117.

19. Jung C-H, Kim B-Y, Kim C-H, Kang S-K, Jung S-H, Mok J-O. Association of serum adipocytokine levels with cardiac autonomic neuropathy in type 2 diabetic patients. Cardiovasc Diabetol. 2012;11(1):24.

20. El Dayem SMA, Battah AA, El Bohy AEM, El Shehaby A, El Ghaffar EA. Relationship of plasma level of chemerin and vaspin to early atherosclerotic changes and cardiac autonomic neuropathy in adolescent type 1 diabetic patients. J Pediatr Endocrinol Metab. 2015;28(3-4):265-73.

21. Grundy SM, Cleeman JI, Daniels SR, Donato KA, Eckel RH, Franklin BA, Gordon DJ, Krauss RM, Savage PJ, Smith SC Jr. Diagnosis and management of the metabolic syndrome: an American Heart Association/National Heart, Lung, and Blood Institute scientific statement. Circulation. 2005;112(17):2735-52.

22. Lin W, Lee L, Chen C, Lo H, Hsia H, Liu I, Lin R, Shau W, Huang K. Optimal cut-off values for obesity: using simple anthropometric indices to predict cardiovascular risk factors in Taiwan. Int $\mathrm{J}$ Obes Relat Metab Disord. 2002;26(9):1232-8. 
23. Yeh W-C, Tsao Y-C, Li W-C, Tzeng I-S, Chen L-S, Chen J-Y. Elevated triglyceride-to-HDL cholesterol ratio is an indicator for insulin resistance in middle-aged and elderly Taiwanese population: a crosssectional study. Lipids Health Dis. 2019;18(1):176.

24. Ma Y-C, Zuo L, Chen J-H, Luo Q, Yu X-Q, Li Y, Xu J-S, Huang S-M, Wang L-N, Huang W. Modified glomerular filtration rate estimating equation for Chinese patients with chronic kidney disease. J Am Soc Nephrol. 2006;17(10):2937-44.

25. Chiu W-C, Lai Y-R, Cheng B-C, Huang C-C, Chen J-F, Lu C-H. HbA1C Variability Is Strongly Associated with Development of Macroalbuminuria in Normal or Microalbuminuria in Patients with Type 2 Diabetes Mellitus: A Six-Year Follow-Up Study. Biomed Res Int. 2020;2020:7462158.

26. Lu C-H, Lin H-C, Huang C-C, Lin W-C, Chen H-L, Chang H-W, Friedman M, Chen CT, Tsai N-W, Wang HC. Increased circulating endothelial progenitor cells and anti-oxidant capacity in obstructive sleep apnea after surgical treatment. Clin Chim Acta. 2015;448:1-7.

27. Wang H-C, Lin W-C, Lin Y-J, Rau C-S, Lee T-H, Chang W-N, Tsai N-W, Cheng B-C, Kung C-T, Lu C-H. The association between serum adhesion molecules and outcome in acute spontaneous intracerebral hemorrhage. Crit Care. 2011;15(6):R284.

28. Low PA. Testing the autonomic nervous system. Semin Neurol. 2003;23(4):407-21.

29. Low PA: Composite autonomic scoring scale for laboratory quantification of generalized autonomic failure. Mayo Clin Proc. 1993;68(8):748 - 52.

30. Huang C-C, Lee J-J, Lin T-K, Tsai N-W, Huang C-R, Chen S-F, Lu C-H, Liu R-T. Diabetic retinopathy is strongly predictive of cardiovascular autonomic neuropathy in type 2 diabetes. $J$ Diabetes Res. 2016;2016:6090749.

31. Ewing D, Clarke B. Diagnosis and management of diabetic autonomic neuropathy. Br Med J (Clin Res Ed). 1982;285(6346):916-8.

32. Malberg H, Wessel N, Hasart A, Osterziel K-J, Voss A. Advanced analysis of spontaneous baroreflex sensitivity, blood pressure and heart rate variability in patients with dilated cardiomyopathy. Clin Sci (Lond). 2002;102(4):465-73.

33. Electrophysiology, TFotESoCtNASoP. Heart rate variability: standards of measurement, physiological interpretation, and clinical use. Circulation. 1996;93(5):1043-65.

34. Alexopoulos N, Katritsis D, Raggi P. Visceral adipose tissue as a source of inflammation and promoter of atherosclerosis. Atherosclerosis. 2014;233(1):104-12.

35. Sader S, Nian M, Liu P: Leptin: a novel link between obesity, diabetes, cardiovascular risk, and ventricular hypertrophy. Circulation. 2003 12;108(6):644-6.

36. Berg AH, Scherer PE. Adipose tissue, inflammation, and cardiovascular disease. Circ Res. 2005;96(9):939-49.

37. Sanchez-Margalet V, Martin-Romero C, Santos-Alvarez J, Goberna R, Najib S, Gonzalez-Yanes C. Role of leptin as an immunomodulator of blood mononuclear cells: mechanisms of action. Clin Exp Immunol. 2003;133(1):11-9. 
38. Halle M, Persson P. Role of leptin and leptin receptor in inflammation. Am J Physiol Regul Integr Comp Physiol. 2003;284(3):R760-2.

39. Atkinson LL, Fischer MA, Lopaschuk GD. Leptin activates cardiac fatty acid oxidation independent of changes in the AMP-activated protein kinase-acetyl-CoA carboxylase-malonyl-CoA axis. J Biol Chem. 2002;277(33):29424-30.

40. Sinha R, Dufour S, Petersen KF, LeBon V, Enoksson S, Ma Y-Z, Savoye M, Rothman DL, Shulman GI, Caprio S. Assessment of skeletal muscle triglyceride content by $1 \mathrm{H}$ nuclear magnetic resonance spectroscopy in lean and obese adolescents: relationships to insulin sensitivity, total body fat, and central adiposity. Diabetes. 2002;51(4):1022-7.

41. Tatti P, Masselli L, Buonanno A, Di Mauro P, Strollo F. Leptin levels in diabetic and nondiabetic subjects. Endocrine. 2001;15(3):305-8.

42. Soliman AT, Omar M, Assem HM, Nasr IS, Rizk MM, El Matary W, El Alaily RK. Serum leptin concentrations in children with type 1 diabetes mellitus: relationship to body mass index, insulin dose, and glycemic control. Metabolism. 2002;51(3):292-6.

43. Wang G, Liu X, Christoffel KK, Zhang S, Wang B, Liu R, Li Z, Liu X, Brickman WJ, Zimmerman D. Prediabetes is not all about obesity: association between plasma leptin and prediabetes in lean rural Chinese adults. Eur J Endocrinol. 2010;163(2):243-9.

44. Simonds SE, Cowley MA: Hypertension in obesity: is leptin the culprit? Trends Neurosci. 2013, 36(2):121-132.

45. Porzionato A, Rucinski M, Macchi V, Stecco C, Castagliuolo I, Malendowicz LK, De Caro R. Expression of leptin and leptin receptor isoforms in the rat and human carotid body. Brain Res. 2011;1385:5667.

46. Shin M-K, Eraso CC, Mu Y-P, Gu C, Yeung BH, Kim LJ, Liu X-R, Wu Z-J, Paudel O, Pichard LE. Leptin induces hypertension acting on transient receptor potential melastatin 7 channel in the carotid body. Circ Res. 2019;125(11):989-1002.

47. Conde SV, Ribeiro MJ, Melo BF, Guarino MP, Sacramento JF. Insulin resistance: a new consequence of altered carotid body chemoreflex? J Physiol. 2017;595(1):31-41.

48. Rolim L, Sá J, Chacra AR, Dib SA. Diabetic cardiovascular autonomic neuropathy: risk factors, clinical impact and early diagnosis. Arq Bras Cardiol. 2008;90(4):e24-31.

49. Dafaalla MD, Nimir MN, Mohammed MI, Ali OA, Hussein A: Risk factors of diabetic cardiac autonomic neuropathy in patients with type 1 diabetes mellitus: a meta-analysis. Open Heart 2016, 3(2):e000336.

50. Lai Y-R, Huang C-C, Chiu W-C, Liu R-T, Tsai N-W, Wang H-C, Lin W-C, Cheng B-C, Su Y-J, Su C-M. $\mathrm{HbA} 1 \mathrm{C}$ variability is strongly associated with the severity of cardiovascular autonomic neuropathy in patients with Type 2 diabetes after longer diabetes duration. Front Neurosci. 2019;13:458.

51. Ewing DJ, Martyn CN, Young RJ, Clarke BF. The value of cardiovascular autonomic function tests: 10 years experience in diabetes. Diabetes Care. 1985;8(5):491-8. 
52. Sahebkar A, Giua R, Pedone C. Impact of statin therapy on plasma leptin concentrations: a systematic review and meta-analysis of randomized placebo-controlled trials. Br J Clin Pharmacol. 2016;82(6):1674-84.

53. Bravo PE, Morse S, Borne DM, Aguilar EA, Reisin E. Leptin and hypertension in obesity. Vasc Health Risk Manag. 2006;2(2):163.

54. Kurniawan LB, Bahrun U, Hatta M, Arif M. Body mass, total body fat percentage, and visceral fat level predict insulin resistance better than waist circumference and body mass index in healthy young male adults in Indonesia. J Clin Med. 2018;7(5):96.

55. Cho D-H, Kim M-N, Joo HJ, Shim WJ, Lim D-S, Park S-M. Visceral obesity, but not central obesity, is associated with cardiac remodeling in subjects with suspected metabolic syndrome. Nutr Metab Cardiovasc Dis. 2019;29(4):360-6.

56. You T, Nicklas BJ. Effects of exercise on adipokines and the metabolic syndrome. Curr Diab Rep. 2008;8(1):7-11.

57. Gæde P, Vedel P, Parving H-H, Pedersen O. Intensified multifactorial intervention in patients with type 2 diabetes mellitus and microalbuminuria: the Steno type 2 randomised study. Lancet. 1999;353(9153):617-22.

58. DiabetesControl, Group CTR. The effect of intensive diabetes therapy on measures of autonomic nervous system function in the Diabetes Control and Complications Trial (DCCT). Diabetologia. 1998;41(4):416-23.

\section{Figures}

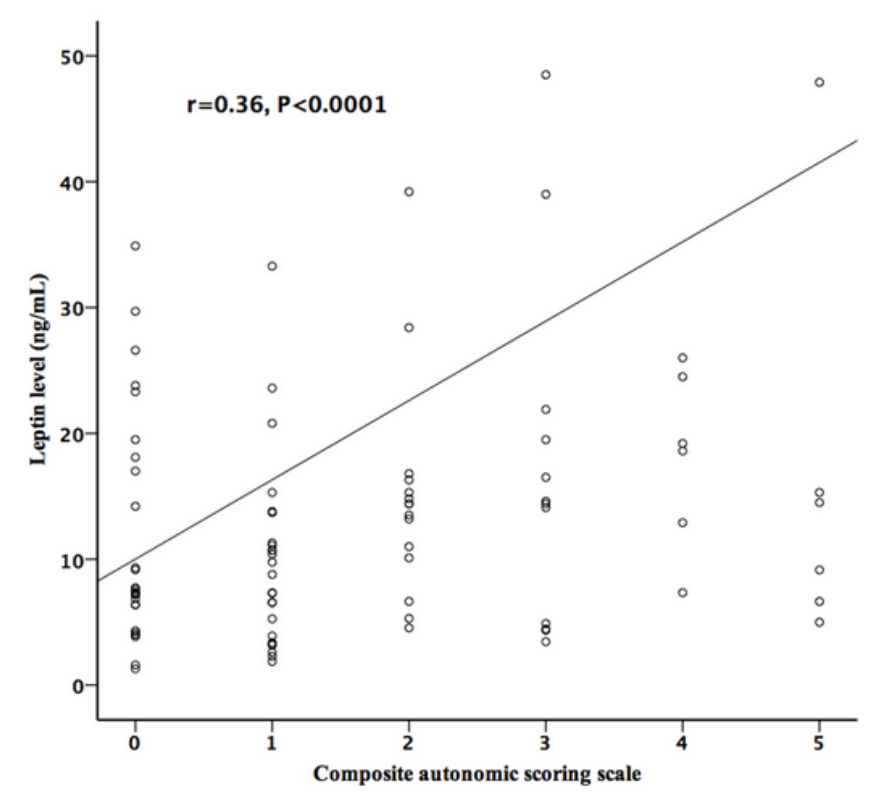

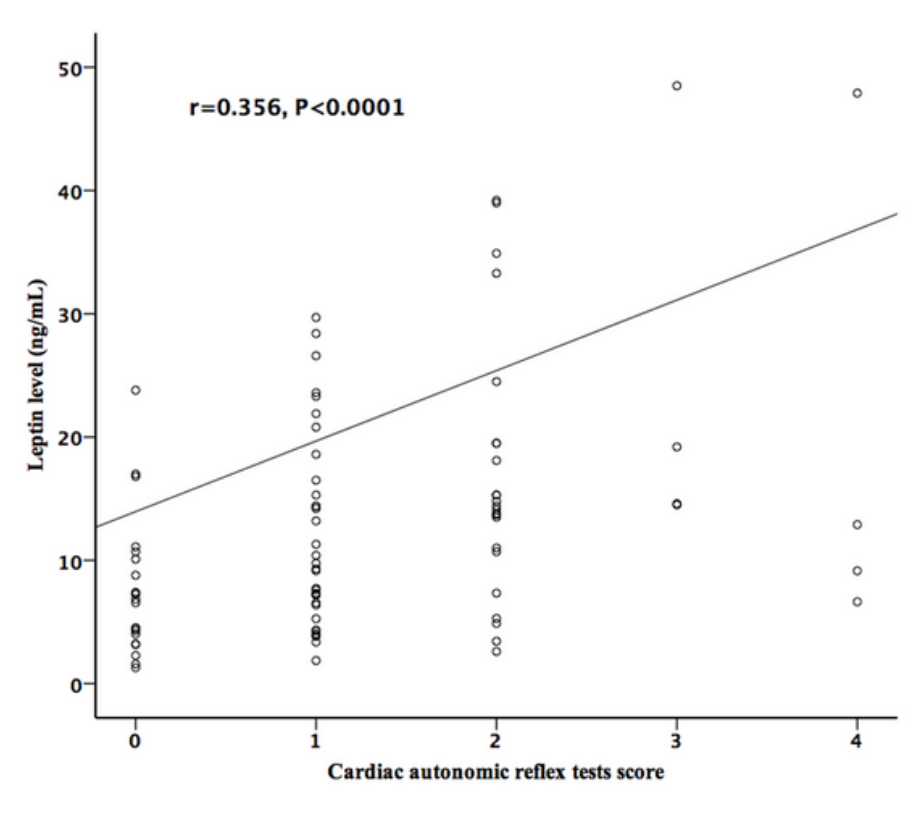

B 


\section{Figure 1}

Relationship between leptin level and composite autonomic scoring scale composite autonomic scoring scale) (Figure 1 A) $(r=0.36, P<0.0001)$ and cardiac autonomic reflex tests score (Figure 1B) $(r=0.356$, $\mathrm{P}<0.0001)$ in patients with type 2 diabetes and prediabetes 